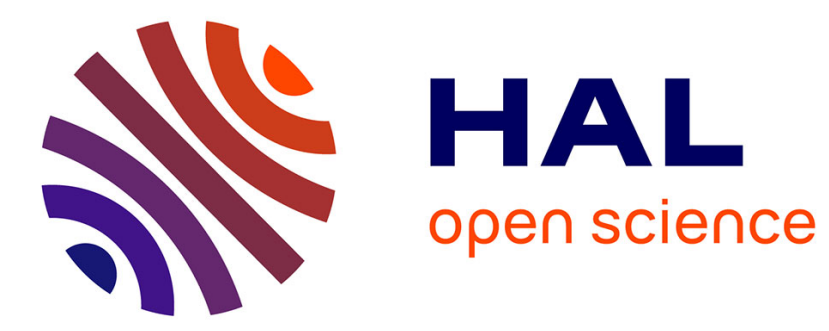

\title{
Targeted fully 3D Monte Carlo reconstruction in SPECT
}

\author{
Z. El Bitar, Y. Petegnief, David R.C. Hill, Vincent Breton, I. Buvat
}

\section{- To cite this version:}

Z. El Bitar, Y. Petegnief, David R.C. Hill, Vincent Breton, I. Buvat. Targeted fully 3D Monte Carlo reconstruction in SPECT. 2006 IEEE Nuclear Science Symposium (NSS), Medical Imaging Conference (MIC) And 15th International Room Temperature Semiconductor Detector Workshop, Oct 2006, San Diego, United States. in2p3-00133773

\section{HAL Id: in2p3-00133773 https://hal.in2p3.fr/in2p3-00133773}

Submitted on 27 Feb 2007

HAL is a multi-disciplinary open access archive for the deposit and dissemination of scientific research documents, whether they are published or not. The documents may come from teaching and research institutions in France or abroad, or from public or private research centers.
L'archive ouverte pluridisciplinaire HAL, est destinée au dépôt et à la diffusion de documents scientifiques de niveau recherche, publiés ou non, émanant des établissements d'enseignement et de recherche français ou étrangers, des laboratoires publics ou privés. 


\title{
Targeted Fully 3D Monte Carlo Reconstruction in SPECT
}

\author{
Ziad El Bitar, Yolande Petegnief, David Hill, Vincent Breton, Irène Buvat
}

\begin{abstract}
Fully 3D Monte-Carlo (F3DMC) reconstruction consists in calculating a fully 3D object-specific system matrix using Monte-Carlo simulations and inverting it using an iterative approach. To reduce the large amount of disk space required by this approach, we derived a targeted F3DMC approach (TF3DMC) in which the volume to be reconstructed is irregularly sampled, so that pre-identified functional regions of interest are reconstructed using fine sampling while regions with non-specific activity or without any particular interest are coarsely sampled. This method was assessed using simulated and real SPECT data of a phantom filled with Tc99m. The GATE Monte-Carlo simulator was considered to simulate the phantom data and to calculate the system matrices needed for the reconstruction of the simulated and of the real SPECT data. Activity ratios measured in TF3DMC images were compared with those measured on F3DMC and OSEM images corrected for scatter, attenuation and detector response function. TF3DLMC yielded errors less than $10 \%$ in activity ratio estimates in hot regions, while errors with quantitative OSEM were between $-21 \%$ and $-3 \%$. The space needed to store the system matrix was divided by a factor from 3.5 to 9.4 compared to F3DMC, for similar or even better accuracy in activity ratio estimates. These results suggest that TF3DMC can be made practical and outperforms F3DMC and OSEM in terms of quantitative accuracy.
\end{abstract}

\section{INTRODUCTION}

$\mathrm{T}_{\mathrm{s}}$ OMOGRAPHIC reconstruction consists in inverting a system matrix describing the imaging process. Ideally, in Single Photon Emission Computed Tomography (SPECT), this system matrix should model the detector response to a specific activity distribution, hence include a model of the geometric detector response together with a model of the object under investigation. Fully 3D Monte-Carlo (F3DMC) reconstruction is a recently proposed approach [1] complying with these two constraints, i.e. in which the system matrix is calculated using Monte-Carlo simulations accounting for the detector response and the patient characteristics. This matrix is then inverted using an iterative approach to complete

Manuscript received November 17, 2006.

Ziad El Bitar and Vincent Breton are with the Laboratory of Corpuscular Physics, Université Blaise Pascal, IN2P3/CNRS, 24 avenue des Landais, 63 177 Aubière Cedex, France (e-mail: elbitar@clermont.in2p3.fr)

Y. Petegnief is with the Laboratory of Positron Imaging, Paris 6 University, Tenon Hospital, Paris, France

D. Hill is with the Laboratory of Informatics, Modeling and System Optimization (LIMOS), Aubière, France

I. Buvat is with UMR 678 INSERM - UPMC, CHU Pitié-Salpêtrière, Paris, France. reconstruction, yielding to images corrected for all patientdependent and detector-dependent biases [1]. When accounting for 3D phenomena introducing biases such as scatter and detector response function, the system matrix gets extremely large (e.g. $128^{6}$ elements in SPECT), which makes the method impractical. To reduce the large amount of disk space required by this approach, we propose a targeted F3DMC (TF3DMC) approach in which the volume to be reconstructed is irregularly sampled, so that pre-identified functional regions of interest are reconstructed using fine sampling while regions with non-specific activity or without any particular interest are coarsely sampled. This study describes the TF3DMC method and compares its performance, in terms of quantitative accuracy, to a F3DMC and conventional quantitative OSEM reconstruction, using simulated and real phantom data.

\section{MATERIALS AND METHODS}

\section{A. Fully 3D Monte-Carlo (F3DMC) reconstruction}

The SPECT reconstruction problem can be written as $\mathrm{p}=\mathrm{Rf}$, where $\mathrm{p}$ is a column vector with $\mathrm{P} \times \mathrm{N}^{2}$ elements - assuming $\mathrm{P}$ projections of $\mathrm{N} \times \mathrm{N}$ pixels are acquired, $\mathrm{f}$ is a column vector of $\mathrm{N}^{3}$ elements - assuming $\mathrm{N}$ transaxial slices $\mathrm{N} \times \mathrm{N}$ are to be estimated, and $\mathrm{R}$ is a $\left(\mathrm{PN}^{2}, \mathrm{~N}^{3}\right)$ fully $3 \mathrm{D}$ system matrix. An element $r_{i j}$ of matrix $R$ represents the probability that a photon emitted in voxel $\mathrm{j}$ be detected in projection pixel $\mathrm{i}$. In F3DMC [1], this system matrix is calculated using Monte-Carlo simulations, given the geometry and attenuation properties of the object and a detector model. The inversion of the system matrix is then performed using the MLEM iterative method.

\section{B. Targeted Fully 3D Monte-Carlo reconstruction (TF3DMC)}

To reduce the impractical size of the system matrix, targeted F3DMC (TF3DMC) samples the volume to be reconstructed using only a small number of functional regions previously identified from a conventional reconstruction, instead of reconstructing a fully voxelized volume $\mathrm{f}$ using a constant voxel size (typically $4 \mathrm{~mm} \times 4 \mathrm{~mm} \times 4 \mathrm{~mm}$ in SPECT). The functional regions of interest are reconstructed using a conventional fine sampling, while only a single value of activity per functional region of no interest is estimated. Activity within the so called "hybrid volume" (partly finely 
sampled and partly coarsely sampled) is estimated by inverting $\mathrm{p}=\mathrm{Rf}$, where $\mathrm{R}$ is now a $\left(\mathrm{PN}^{2}, \mathrm{~F}\right)$ system matrix and $\mathrm{F}$ is the number of values to be estimated (much smaller than the total number of voxels). Similar to F3DMC, R is calculated using Monte-Carlo simulations, given the geometry and attenuation properties of the object and a detector model, and the inversion of $\mathrm{R}$ is performed using MLEM.

\section{Phantoms}

TF3DMC was assessed using a Monte-Carlo simulation performed using the GATE toolkit [2] and a real acquisition of a cylindrical $\mathrm{Tc}^{99 \mathrm{~m}}$ phantom. An AXIS Philips gamma camera fitted with a low energy high resolution collimator was considered in both cases.

The simulated phantom was a cylinder $(10 \mathrm{~cm}$ high, $10 \mathrm{~cm}$ in diameter) including 6 rods (4.8 to $12.7 \mathrm{~mm}$ in diameter) with rod-to-background $\mathrm{Tc}^{99 \mathrm{~m}}$ activity ratios of 0 (largest rod) or 4 (all other rods). The real phantom was also a cylinder (21.1 $\mathrm{cm}$ high, $22 \mathrm{~cm}$ in diameter) including 7 spheres (6.2 to $19 \mathrm{~mm}$ in diameter) with sphere-to-background activity ratios of 0 (largest sphere) or 4 (all other spheres).

\section{Reconstruction and data processing}

Images were reconstructed using F3DMC, TF3DMC and using OSEM. In TF3DMC, for the simulated data, 7 functional regions were considered: one for each rod, which were reconstructed using a fine sampling (3.125 mm x 3.125 $\mathrm{mm} \times 3.125 \mathrm{~mm}$ ), and one for the rest of the phantom, which was reconstructed as a single value. For the real data, 2 functional regions were considered: 1 corresponding to all slices including the spheres, which was reconstructed using a fine sampling $(6.17 \mathrm{~mm} \times 6.17 \mathrm{~mm} \times 6.17 \mathrm{~mm})$, and one corresponding to all other slices, which was reconstructed as a single value. For F3DMC and TF3DMC, iterations were stopped when the sum of the squared differences between the estimated projection bins and the measured projection bins did not change by more than $10^{-3}$ from an iteration to the next (which corresponded to about 200 iterations). Data were also reconstructed using OSEM including corrections for attenuation (modelled in OSEM for the simulated data, and using the iterative Chang algorithm for the real data) and scatter (Jaszczak scatter subtraction). In addition, the simulated data were corrected for detector response function by modelling this response into the system matrix. The number of subsets times the number of iterations was chosen so as to obtain stable rod (or sphere) to background activity ratios without excessive noise.

To assess quantitative accuracy, activity ratios between rods (or spheres) and background were measured in the F3DMC, TF3DMC and OSEM images, by considering identical regions of interest for all reconstructed images. For the simulated data, regions matching exactly the contours of the rods were used, while for the real data, regions of the exact sphere sizes were considered, and the background region was located in the slices including no sphere, far from the border of the phantom.

The signal to noise ratio (SNR) was calculated for real data. using 30 noisy replications of the acquired projections. The mean number of counts in the projections and associated standard deviation were $11045646 \pm 1364$. Each noisy replication was reconstructed using the three reconstruction methods: OSEM, F3DMC and TF3DMC, and the mean and standard deviation images of the 30 reconstructed image sets were calculated for each reconstruction method. For each reconstruction method, SNR was defined as the number of counts within a volume of interest calculated in the mean image over the number of counts within the same volume of interest calculated in the standard deviation image. The volume of interest used to estimate the SNR was a $9 \mathrm{~mm}$ in diameter spherical volume drawn in the slices containing the spheres of interest but far from these spheres.

To reduce the time needed for the calculation of the system matrix, a large number of simulations were run on the European Computing grid EGEE [3]. 74 and 216 billion photons were simulated to calculate the system matrix corresponding to the simulated phantom and to the real phantom respectively.

\section{RESULTS AND DISCUSSION}

\section{A. Simulations}

Figure 1 shows the ideal image and the reconstructed images of the simulated phantom using the three reconstructed methods.

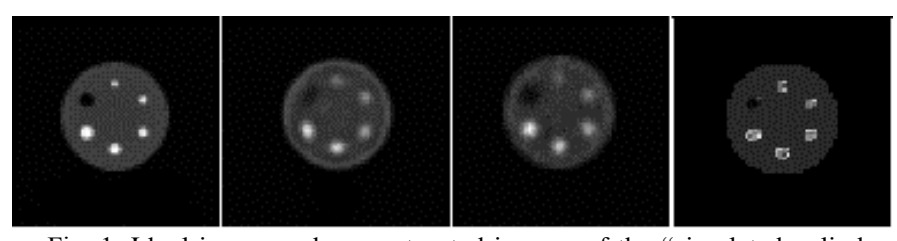

Fig. 1. Ideal image and reconstructed images of the "simulated cylinder phantom" using the three reconstruction approaches.

The errors in activity ratio estimates for the hot rods, with respect to ideal values measured on the emission images, are shown in Table 1. Ideal values are different from the expected 0 and 4 values that were set to define the phantom because of the errors introduced by the sampling of the object on a pixel matrix. Table 1 suggests an improved accuracy of TF3DMC with respect to OSEM. TF3DMC also appears to yield more accurate results than F3DMC, suggesting a faster convergence of TF3DMC with respect to F3DMC.

\begin{tabular}{ccccl} 
Rod diameter & Ideal values & OSEM & TF3DMC & F3DMC \\
\hline $4.8 \mathrm{~mm}$ & 1.92 & $1.61(-16 \%)$ & $1.94(+1 \%)$ & $1.65(-16 \%)$ \\
$6.4 \mathrm{~mm}$ & 2.23 & $1.80(-19 \%)$ & $2.34(+5 \%)$ & $2.01(-11 \%)$ \\
$7.8 \mathrm{~mm}$ & 2.46 & $1.93(-21 \%)$ & $2.46(+1 \%)$ & $2.15(-14 \%)$ \\
$9.5 \mathrm{~mm}$ & 2.54 & $2.47(-3 \%)$ & $2.52(-1 \%)$ & $2.42(-5 \%)$ \\
$11.1 \mathrm{~mm}$ & 2.73 & $2.46(-10 \%)$ & $2.95(+9 \%)$ & $2.66(-2 \%)$ \\
$12.7 \mathrm{~mm}$ & 0.41 & $0.71(+73 \%)$ & $0.47(+14 \%)$ & $0.52(+22 \%)$ \\
\hline
\end{tabular}

Table 1. Rod-to-background activity values for simulated data obtained using the OSEM, the TF3DMC and the F3DMC reconstruction methods.

Figure 2 shows the percent errors in activity ratios with respect to the number of simulated photons simulated to calculate the system matrix for images reconstructed with F3DMC and TF3DMC methods. 


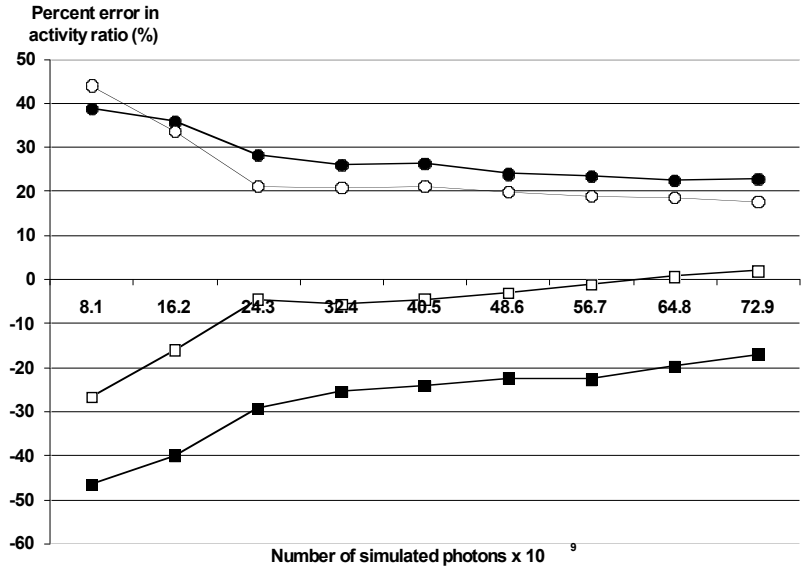

Fig. 2. Percent error in activity concentration ratios with respect to the number of photons simulated for calculation of the system matrix. Circles: $12.7 \mathrm{~mm}$ rod, squares: $4.8 \mathrm{~mm}$ rod. Black symbols: F3DMC reconstruction, Open symbols: TF3DMC reconstruction.

Figure 2 suggests that when reconstruction is performed using TF3DMC, less photons need to be simulated than when reconstructing with $\mathrm{F} 3 \mathrm{DMC}$, to achieve the same level of quantitative accuracy in activity ratio estimates.

\section{B. Real data}

Table 2 presents the errors in activity ratio estimates for the hottest 4 spheres visible on the OSEM images (the smallest two spheres could not be seen in the images), with respect to the ideal values. This table confirms the greater accuracy of TF3DMC with respect to OSEM in real data. On these data, TF3DMC and F3DMC present similar levels of accuracy (i.e., TF3DMC did not converge faster than F3DMC), probably because a larger proportion of the volume to be reconstructed was finely sampled than in the case of the simulated data.

\begin{tabular}{ccccl} 
Rod diameter & Ideal values & OSEM & TF3DMC & F3DMC \\
\cline { 1 - 4 } $9.15 \mathrm{~mm}$ & 1.93 & $1.70(-12 \%)$ & $2.05(+6 \%)$ & $2.05(+6 \%)$ \\
$12.55 \mathrm{~mm}$ & 2.11 & $1.69(-20 \%)$ & $2.12(+1 \%)$ & $2.09(+1 \%)$ \\
$15.6 \mathrm{~mm}$ & 2.31 & $1.90(-18 \%)$ & $2.27(-2 \%)$ & $2.21(-4 \%)$ \\
$18.7 \mathrm{~mm}$ & 2.39 & $2.07(-13 \%)$ & $2.42(+1 \%)$ & $2.40(1 \%)$ \\
$19 \mathrm{~mm}$ & 0.46 & $0.70(+52 \%)$ & $0.68(+48 \%)$ & $0.73(59 \%)$ \\
\hline
\end{tabular}

Table 2. Rod-to-background activity values for the real data obtained using the OSEM, the TF3DMC and the F3DMC reconstruction methods.

Figure 3 shows the change in activity ratios as a function of the number of simulated photons used for calculating the system matrix. This figure suggests that quantification could still be improved in the cold region by further increasing the statistics used to estimate the system matrix. It also shows that the reconstruction converges faster in hot regions than in cold regions.

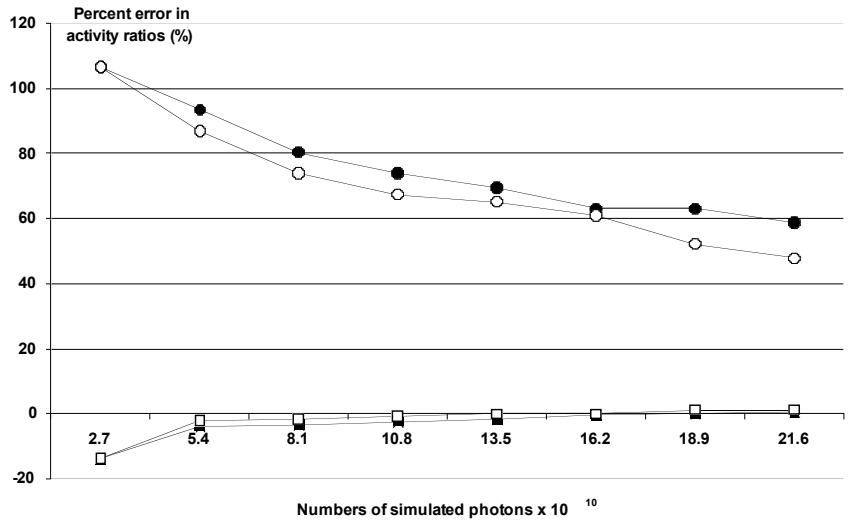

Fig. 3. Percent error in activity ratio with respect to the number of simulated photons. Circles represent the $19 \mathrm{~mm}$ cold sphere, squares represent the 18.7 $\mathrm{mm}$ hot sphere. Black symbols: F3DMC reconstruction, Open symbols: TF3DMC reconstruction. The hot sphere region converges faster than the cold sphere region.

Figure 4 shows the change in the variance of the mean probability for a photon emitted from the center of the real phantom (sphere having a diameter of $4 \mathrm{~cm}$ ) to be detected in the center of the projections (circle having $4 \mathrm{~cm}$ of diameter) with respect to the number of simulated photons used to calculate the system matrix. Convergence does not seem to be achieved yet, although an asymptotic trend can be guessed.

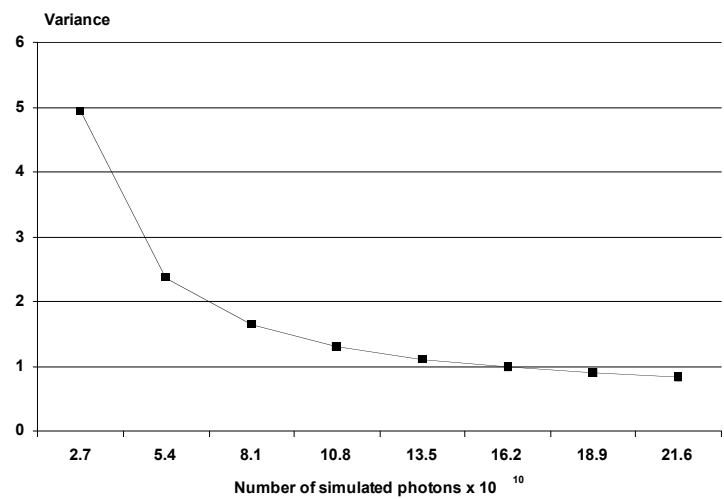

Fig. 4. Convergence rate of the variance of the mean probability for a photon emitted from a sphere located at the centre of the real phantom to be detected in an area in the center of the projections.

Figure 5 shows the variation in the signal to noise ratio in the F3DMC and TF3DMC reconstructed images as a function of the number of simulated photons used for the calculation of the system matrix. 


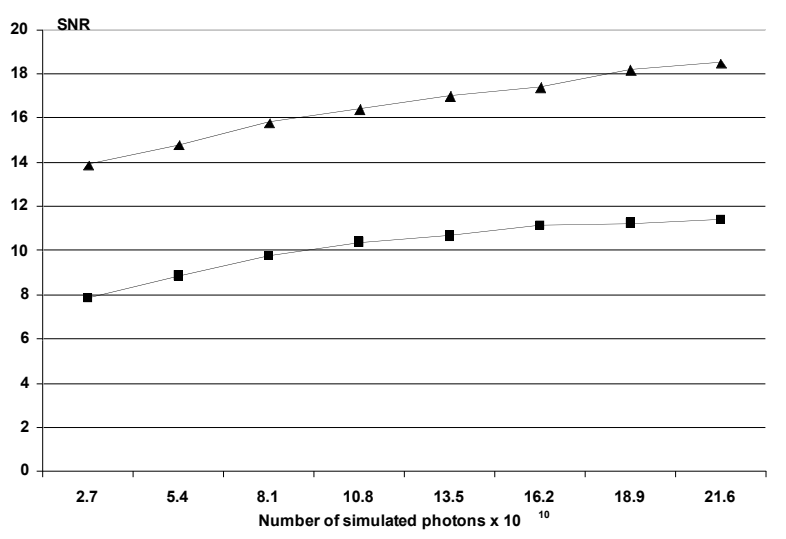

Fig. 5. Signal to noise ratio with respect to the number of simulated photons used for the calculation of the system matrix. Squares: F3DMC reconstruction, Triangles : TF3DMC reconstruction.

In OSEM images, SNR was equal to 8.72 .

As shown in Figure 5, SNR calculated on images reconstructed with F3DMC and TF3DMC methods increased as the number of simulated photons used for the calculation of the system matrix increased. With F3DMC, the SNR was about twice higher than with OSEM when considering the system matrix calculated using 21.6 billion photons.

\section{Disk space requirement}

Figures 6 and 7 show that using TF3DMC reconstruction greatly reduces the disk space needed for the storage of the system matrix.

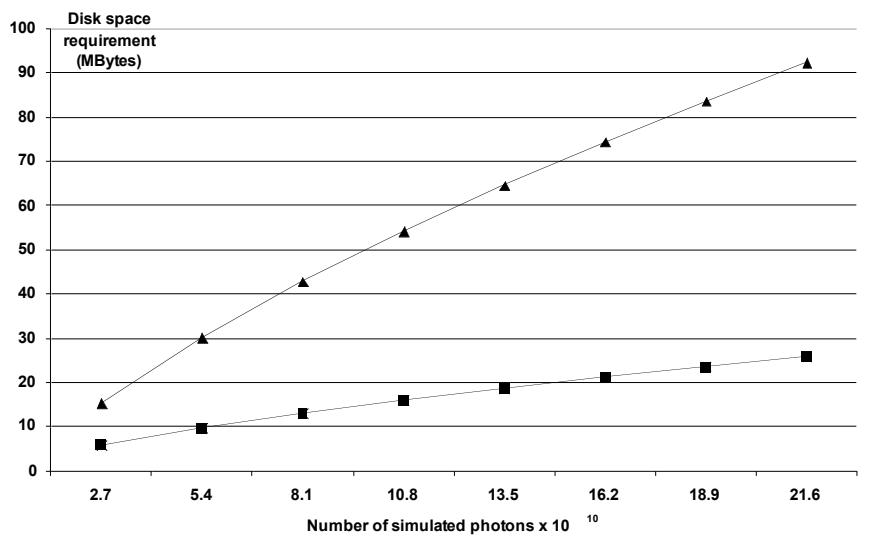

Fig. 6. Disk space requirement for storing the system matrix as a function of the number of simulated photons for the real data. Triangles : F3DMC system matrix. Squares : TF3DMC system matrix.

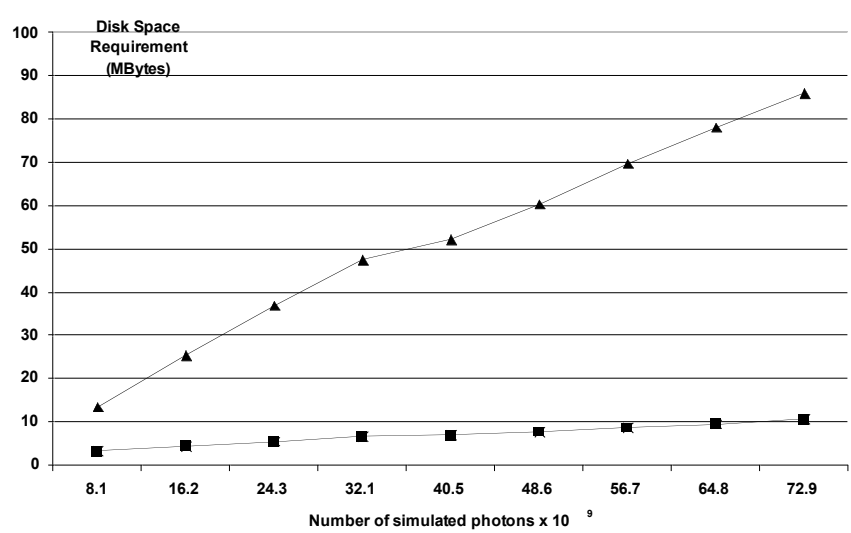

Fig. 6. Disk space requirement for storing the system matrix as a function of the number of simulated photons for the simulated data. Triangles : F3DMC system matrix. Squares : TF3DMC system matrix.

\section{CONCLUSION}

TF3DMC shows promising results for accurate estimation of activity values in pre-selected regions of interest and for the improvement of the signal to noise ratio, while significantly reducing disk space requirement compared to F3DMC.

Properly targeting the functional regions of interest might be feasible from a preliminary conventional reconstruction aiming at identifying either suspicious regions or regions in which accurate quantification is required.

\section{REFERENCES}

[1] Lazaro D, El Bitar Z, Breton V, Hill D, Buvat I. Fully 3D Monte-Carlo reconstruction in SPECT: a feasibility study. Phys Med Biol 50: 37393754, 2005.

[2] S.Jan et al. GATE a simulation toolkit for PET and SPECT.Phys Med Biol.49 (2005) 4543-61

[3] http://www.eu-egee.org/ 